\title{
Using multi-echo simultaneous multi-slice (SMS) EPI to improve functional MRI of the subcortical nuclei of the basal ganglia at ultra-high field (7T)
}

Citation for published version (APA):

Puckett, A. M., Bollmann, S., Poser, B. A., Palmer, J., Barth, M., \& Cunnington, R. (2018). Using multiecho simultaneous multi-slice (SMS) EPI to improve functional MRI of the subcortical nuclei of the basal ganglia at ultra-high field (7T). Neuroimage, 172, 886-895.

https://doi.org/10.1016/j.neuroimage.2017.12.005

Document status and date:

Published: 15/05/2018

DOI:

10.1016/j.neuroimage.2017.12.005

Document Version:

Publisher's PDF, also known as Version of record

Document license:

Taverne

Please check the document version of this publication:

- A submitted manuscript is the version of the article upon submission and before peer-review. There can be important differences between the submitted version and the official published version of record.

People interested in the research are advised to contact the author for the final version of the publication, or visit the DOI to the publisher's website.

- The final author version and the galley proof are versions of the publication after peer review.

- The final published version features the final layout of the paper including the volume, issue and page numbers.

Link to publication

\footnotetext{
General rights rights.

- You may freely distribute the URL identifying the publication in the public portal. please follow below link for the End User Agreement:

www.umlib.nl/taverne-license

Take down policy

If you believe that this document breaches copyright please contact us at:

repository@maastrichtuniversity.nl

providing details and we will investigate your claim.
}

Copyright and moral rights for the publications made accessible in the public portal are retained by the authors and/or other copyright owners and it is a condition of accessing publications that users recognise and abide by the legal requirements associated with these

- Users may download and print one copy of any publication from the public portal for the purpose of private study or research.

- You may not further distribute the material or use it for any profit-making activity or commercial gain

If the publication is distributed under the terms of Article 25fa of the Dutch Copyright Act, indicated by the "Taverne" license above, 


\title{
Using multi-echo simultaneous multi-slice (SMS) EPI to improve functional MRI of the subcortical nuclei of the basal ganglia at ultra-high field (7T)
}

\author{
Alexander M. Puckett ${ }^{\mathrm{a},{ }^{*}, 1}$, Saskia Bollmann ${ }^{\mathrm{b}, 1}$, Benedikt A. Poser ${ }^{\mathrm{c}}$, Jake Palmer ${ }^{\mathrm{d}}$, \\ Markus Barth $^{\mathrm{b}, 1}$, Ross Cunnington ${ }^{\mathrm{a}, \mathrm{d}, 1}$ \\ ${ }^{a}$ Queensland Brain Institute, The University of Queensland, Brisbane, QLD 4072, Australia \\ ${ }^{\mathrm{b}}$ Centre for Advanced Imaging, The University of Queensland, Brisbane, QLD 4072, Australia \\ ${ }^{\mathrm{c}}$ Department of Cognitive Neuroscience, Faculty of Psychology and Neuroscience, Maastricht University, Maastricht, The Netherlands \\ d School of Psychology, The University of Queensland, Brisbane, QLD, 4072, Australia
}

\section{A R T I C L E I N F O}

\section{Keywords:}

Functional MRI

EPI

Simultaneous multi-slice

Multi-echo

Susceptibility artifacts

Signal loss

Echo time

Motor

\begin{abstract}
A B S T R A C T
The nuclei of the basal ganglia pose a special problem for functional MRI, especially at ultra-high field, because T2* variations between different regions result in suboptimal BOLD sensitivity when using gradient-echo echoplanar imaging (EPI). Specifically, the iron-rich lentiform nucleus of the basal ganglia, including the putamen and globus pallidus, suffers from substantial signal loss when imaging is performed using conventional single-echo EPI with echo times optimized for the cortex. Multi-echo EPI acquires several echoes at different echo times for every imaging slice, allowing images to be reconstructed with a weighting of echo times that is optimized individually for each voxel according to the underlying tissue or T2* properties. Here we show that multi-echo simultaneous multi-slice (SMS) EPI can improve functional activation of iron-rich subcortical regions while maintaining sensitivity within cortical areas. Functional imaging during a motor task known to elicit strong activations in the cortex and the subcortex (basal ganglia) was performed to compare the performance of multi-echo SMS EPI to single-echo SMS EPI. Notably within both the caudate nucleus and putamen of the basal ganglia, multi-echo SMS EPI yielded higher tSNR (an average 84\% increase) and CNR (an average 58\% increase), an approximate 3-fold increase in supra-threshold voxels, and higher $t$-values (an average $39 \%$ increase). The degree of improvement in the group level $t$-statistics was negatively correlated to the underlying $\mathrm{T} 2 *$ of the voxels, such that the shorter the $\mathrm{T} 2 *$, as in the iron-rich nuclei of the basal ganglia, the higher the improvement of $t$-values in the activated region.
\end{abstract}

\section{Introduction}

Blood oxygenation level dependent (BOLD) fMRI is most commonly performed using a gradient-echo echo-planar imaging (GE-EPI) sequence at high (3T) or ultra-high field $(\geq 7 \mathrm{~T})$ for enhanced functional sensitivity. In conventional GE-EPI sequences, the functional contrast depends on the chosen echo time (TE). The maximum BOLD contrast occurs at the TE at which the difference in the $2 *$ signal decay of the oxygenated states of hemoglobin is maximal. In a first approximation, this is achieved when matching the TE with the effective transverse relaxation time (T2*) of the underlying tissue (Gati et al., 1997; Triantafyllou et al., 2005). A particular challenge for functional MRI of subcortical nuclei is the significant regional variation in $\mathrm{T} 2 *$ that exists across different brain regions, specifically between cortical and subcortical regions, i.e. T2* in subcortical nuclei is considerably shorter than in cortical regions (Sedlacik et al., 2014; Cohen-Adad et al., 2012; Deistung et al., 2013; Yao et al., 2009).

The significant regional variation in $\mathrm{T} 2 *$ across different brain areas is caused by two effects, i.e. (i) by tissue-intrinsic (microscopic T2 and diffusion) as well as mesoscopic (smaller than voxel size, but larger scale than microscopic) relaxation effects, and (ii) by macroscopic effects from static field inhomogeneities (Yablonskiy, 1998). The macroscopic effects are caused by magnetic susceptibility differences at the interfaces between tissue, air, and bone (Volz et al., 2009) or suboptimal shim settings and can result in signal loss and image distortions. As these macroscopic effects depend on acquisition parameters such as slice-thickness and orientation, they can be partly reduced by optimizing a range of sequence properties in a whole-brain (Weiskopf et al., 2006, 2007; Robinson et al.,

\footnotetext{
* Corresponding author. QBI Building \#79, University of Queensland, St. Lucia, QLD 4072 Australia.

E-mail address: a.puckett@uq.edu.au (A.M. Puckett).

${ }^{1}$ Indicates shared first/senior authorship.
} 
2008) or in a slice-by-slice fashion (Brunheim et al., 2017; Stocker et al., 2006).

While these techniques are well-suited to compensate for large-scale, temporally static, and gradually changing susceptibility gradients in space, they cannot compensate for microscopic and mesoscopic effects caused by the high iron content within the basal ganglia nuclei (Gelman et al., 1999; Ordidge et al., 1994), which lead to a short T2* in these brain regions (Robinson et al., 2004; Schenck, 2003; Peters et al., 2007). The reduced $\mathrm{T} 2 *$ then causes considerable signal loss in EPI images using a single TE optimized for cortical regions (Berger et al., 2016; Koopmans et al., 2011; van der Zwaag et al., 2009; Yacoub et al., 2001) as this TE is usually much longer than the T2* of the basal ganglia nuclei at ultra-high field (Cohen-Adad et al., 2012; Deistung et al., 2013). Hence it is impossible for single-echo EPI to provide an optimal BOLD sensitivity for the entire brain.

Multi-echo fMRI is a technique that has been shown to overcome regional $\mathrm{T} 2 *$ variation and enhance functional sensitivity (Posse et al., 1999). Multi-echo imaging involves collecting multiple sets of echoes at a range of different TEs, which can then be combined to optimize functional sensitivity in a voxel-wise manner depending on the optimal TE for the underlying tissue. Applied to GE-EPI and in combination with parallel imaging, multi-echo EPI has been shown to improve functional sensitivity at both 3T (Poser et al., 2006) and 7T (Poser and Norris, 2009) with larger improvements seen in regions suffering from susceptibility-induced inhomogeneities. Here, we investigate the capabilities of multi-echo imaging to compensate for the mesoscopic, within-voxel inhomogeneities that occur in iron-rich subcortical nuclei such as the basal ganglia while retaining high sensitivity in cortical regions. In this study, we combined the multi-echo readout with a state-of-the-art implementation of a simultaneous-multislice (SMS) acquisition and reconstruction technique to obtain a sub-second volume repetition time (TR) for whole-brain imaging.

\section{Theory}

\section{Multi-echo imaging for fMRI}

Multi-echo EPI is a functional imaging technique that can increase the BOLD sensitivity while simultaneously reducing image distortions and signal loss (Poser and Norris, 2009). In essence, multi-echo imaging involves acquiring multiple echoes in one shot following a single RF pulse. This opens up various possibilities for how to process the data and to exploit the additional information that is obtained by simultaneously acquiring the BOLD signal at different echo times. A straightforward option is to combine the different echoes into a single image time series, which can then be processed and analyzed in a conventional manner just like a single-echo acquisition; this is the path chosen for the present study. The change in T2* due to changes in blood oxygen level can also be investigated directly by fitting a mono-exponential decay to the different echoes (Speck and Hennig, 1998). Another option that has gained interest recently is to use the multi-echo information for automatic denoising with the MEICA technique (Kundu et al., 2017, 2013, 2012). This method attempts to separate 'BOLD like' from 'non-BOLD like' signal components (and filter out the latter) by performing an ICA across the spatial concatenation of the multiple echoes and then characterizing their TE dependence as T2* induced (linear TE dependence, i.e. BOLD like) or $\mathrm{S}_{0}$ induced (no TE dependence, i.e. artifact like). However, the focus of this study was on BOLD sensitivity, and the ability of multi-echo EPI to provide more uniform sensitivity across different underlying T2* values; hence, we chose a form of weighted echo combination as described below.

Echo combination using a weighted summation has been shown to maximize the contrast-to-noise-ratio (CNR) by matching the weights applied to images of different echo times to the bell-shaped BOLD sensitivity curve (Posse et al., 1999). However, this requires the accurate knowledge of the $\mathrm{T} 2 *$ values at each voxel location. An alternative strategy has been proposed by Poser et al. (2006), in which the different echoes are weighted directly by their measured CNR. To avoid confounds through task related signal changes, the tSNR needs to be estimated from additional calibration scans. However, a simple average across the different TE images outperformed the CNR-weighted average in regions with short T2* (Poser and Norris, 2009), and a recent study by Kettinger et al. (2016) has shown that all combination schemes perform equally well. Here, we used the temporal average of the time series (AVG) instead of the tSNR. The benefit of this weighting scheme is that the weights can be directly estimated from the data and no additional calibration scans or model assumptions are required. Importantly, changes in the signal due to BOLD fluctuations (i.e. due to task or resting-state fluctuations) do not influence the weights as they primarily affect the estimated variance, but not the average.

$w_{n}=\frac{A V G_{n} \cdot T E_{n}}{\sum A V G_{n} \cdot T E_{n}}$

One potential limitation of multi-echo EPI is the prolonged readout time, which is especially challenging for 7T-applications due to the short $\mathrm{T} 2 *$ values in grey matter and subcortical nuclei in comparison to lower field strength (Peters et al., 2007; Yao et al., 2009). However, parallel imaging can be used to drastically reduce the number of phase encoding steps, which in turn reduces the EPI readout time and simultaneously increases the bandwidth in phase encoding direction to reduce image distortions and blurring (Poser et al., 2006). This has enabled the successful applications of multi-echo EPI at 7T to reduce artifacts and enhance functional contrast (Poser and Norris, 2009). In a separate development, SMS EPI (Feinberg et al., 2010; Setsompop et al., 2012) based on earlier work by Larkman et al. (2001) and Breuer et al. (2005) has brought the capability to significantly reduce the TR by acquiring multiple slices at once; for a review on this topic see Barth et al. (2016). In this study, we combine both the ME-EPI and SMS-EPI techniques, which allows us to acquire multi-echo EPI with whole-brain coverage at a temporal resolution of well below one second TR.

\section{Motor cortex and basal ganglia}

When performing complex motor movements, activity is elicited in a wide network of areas including cortical motor areas and subcortical nuclei of the basal ganglia and thalamus (Bednark et al., 2015; Cunnington et al., 2002; Lehericy et al., 2006). These subcortical nuclei within the basal ganglia are known to be high in iron content. Early postmortem histological evidence showed that non-heme iron levels vary throughout the brain with the highest concentration consistently found within the basal ganglia, particularly within the lentiform nucleus that contains regions of the putamen and globus pallidus (Drayer et al., 1986; Hallgren and Sourander, 1958). These early findings have been confirmed in-vivo through recently developed MRI techniques that form susceptibility-weighted images sensitive to iron levels (Deistung et al., 2013; Haacke et al., 2004, 2005; Schafer et al., 2012; Schweser et al., 2011). While the high iron content of the basal ganglia can be exploited for anatomical imaging to improve delineation of structural boundaries (Abosch et al., 2010), it is highly detrimental for the functional contrast necessary for fMRI. This is particularly problematic at high magnetic field strengths as the signal decays even more rapidly resulting in shorter T2* and very little signal remaining at typically used TEs (Schenck, 2003). Multi-echo EPI sequences may therefore be ideally suited to whole-brain fMRI of complex motor movements at 7T, to improve the concurrent imaging of cortical and subcortical areas that differ in underlying T2* values and thereby to compensate for the signal loss typical seen in fMRI of the basal ganglia.

\section{Methods}

To test whether multi-echo EPI can improve functional imaging of 
cortical and subcortical areas over single-echo EPI for a complex motor task, we performed whole-brain fMRI during finger motor movements known to elicit coordinated activity in primary motor cortex (M1), supplementary motor cortex (SMA), and the basal ganglia. Data were collected using both single-echo and multi-echo sequences for each participant to allow direct comparison between MRI sequence types.

\section{Participants}

$\mathrm{N}=10$ right-handed participants (7 female) in the age range of 21-30 years $(\mathrm{M}=25.1, \mathrm{SD}=3.6$ years $)$ with no history of neurological or psychiatric diseases participated in this study. The experiment was conducted with the understanding and written consent of each subject and was approved by the local ethics committee and in accordance with national guidelines.

\section{Experimental design}

Participants performed motor movements in response to visual cues while inside the MRI scanner (Fig. 1). The visual stimuli were presented on a projector screen at the back of the scanner, viewed from a mirror mounted on the head coil. There were two experimental conditions, simple and complex finger-movements, which both involved making four-finger motor movements by pressing buttons in sequence on a fourbutton response box with the index, middle, ring, and little fingers of the right hand. Previous work has shown that this movement task elicits focal clusters of differential activity in cortical motor areas and within the basal ganglia (Bednark et al., 2015). The rationale was to therefore use these two conditions to localize and then examine the responses in voxels simultaneously activated in both cortex and subcortex under multi-echo versus single-echo imaging sequences.

The motor responses in both conditions (i.e. simple and complex) were self-paced and differed only in the duration of button presses. The stimulus for the simple movement condition (Fig. 1A) consisted of four equal sized and evenly spaced dashes. Subjects made four consistent, medium length button presses with the same stimulus presented for each trial within a simple block. For the complex movement condition (Fig. 1B), the stimulus consisted of dots and dashes indicating button presses of different durations, in a Morse code style. Dots represented a short button press while dashes represented a long button press, with a combination of two short and two long presses per presentation. Therefore, there were six possible combinations of short and long button presses for the complex condition. A single trial of either the simple or complex movement condition involved the visual cue being presented for $2500 \mathrm{~ms}$, followed by a fixation cross that was presented for another $500 \mathrm{~ms}$, as shown in Figure 1. Subjects were instructed to complete the movement before the next presentation of the fixation cross but to remain consistent, taking up the entire available time to complete the movement.

The fMRI paradigm followed a block design consisting of three types of blocks: simple movement, complex movement, and rest (Fig. 1C). Each movement block contained six single trials (3000 ms trial length), in which participants performed either the simple or complex movement, resulting in a total block length of $18 \mathrm{~s}$. In every complex movement block, each of the six possible combination of short and long button presses was presented once. Between complex movement blocks, the order of presentation of the different combinations was randomized. In the simple movement block, participants performed the simple movement repeatedly. In the rest block, participants were instructed to
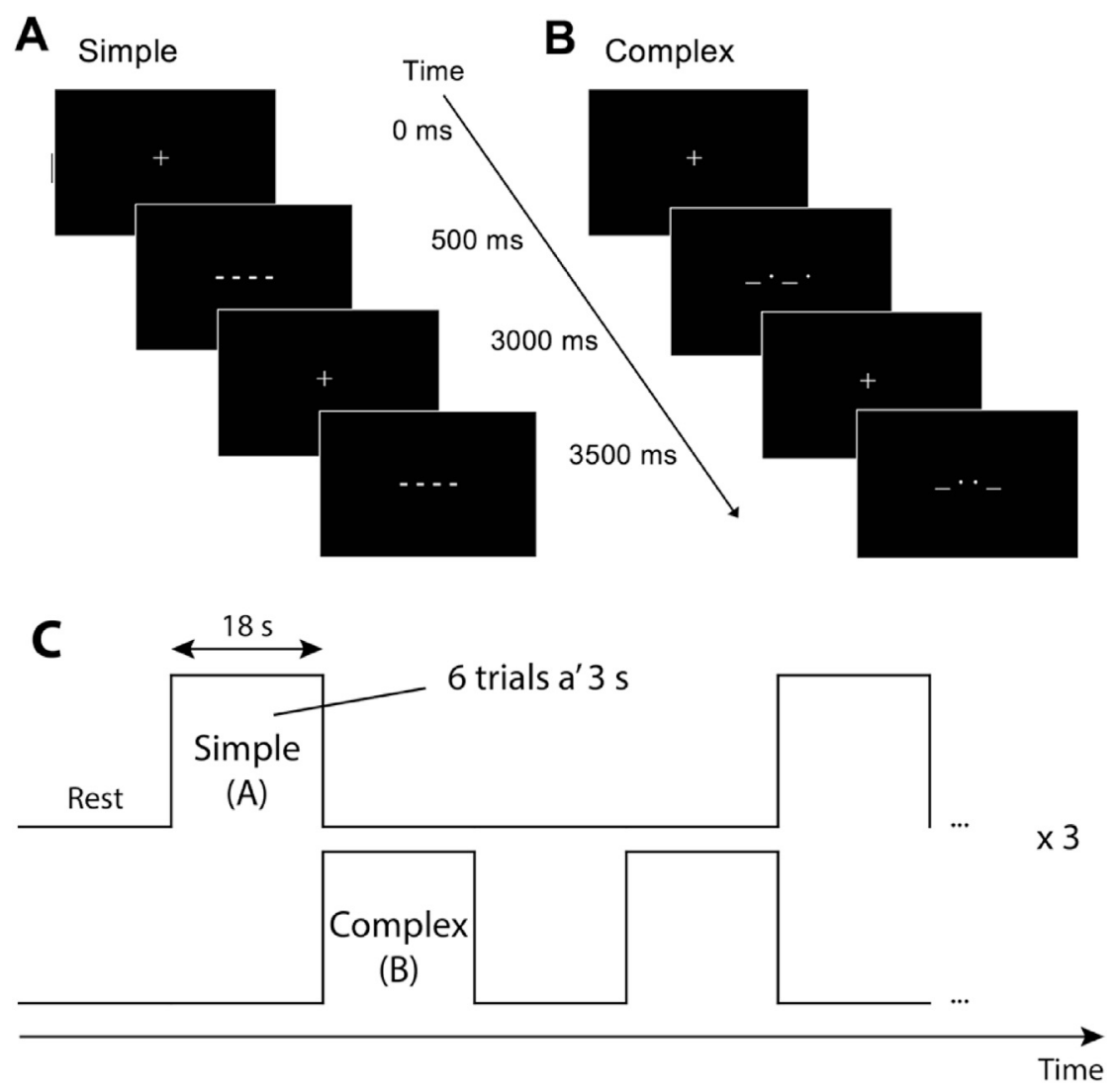

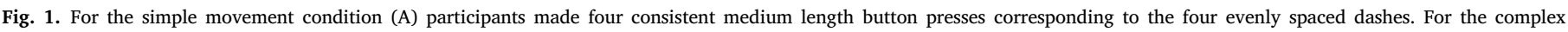

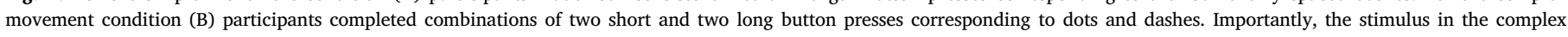

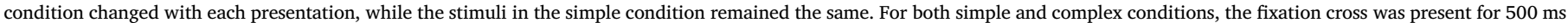

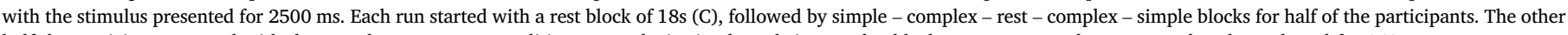
half the participants started with the complex movement condition. In total, six simple and six complex blocks were presented per run, and each run lasted for $342 \mathrm{~s}$. 
passively view the fixation cross.

For all participants, each fMRI run started with a rest block (Fig. 1C). The order of the first presentation of each of the two following movement conditions was then counterbalanced between participants. Specifically, half of the participants followed the order of rest - simple - complex rest - complex - simple repeated, while the other half followed the order of rest - complex - simple - rest - simple - complex repeated. An extra rest block was also included following the final movement condition to ensure the BOLD signal associated with the movement was acquired. In total, each movement condition was presented six times and a full run lasted of $342 \mathrm{~s}$. Two functional runs were collected using each sequence (i.e. single-echo and multi-echo) within the scan session, and the order was counterbalanced with respect to acquisition sequence.

Before beginning the experiment, participants completed a training run. The training was sufficiently short to ensure the complex movement sequences were not learned, but participants were able to become familiar with the visual cues, button responses, and requirements of the task. This training took place either outside the scanner on a standard desktop computer and keyboard or in the scanner before the first functional scan.

\section{Magnetic resonance imaging data acquisition}

All images were acquired on a whole-body 7T Siemens Magnetom MRI scanner (Siemens Healthcare, Erlangen, Germany) with a 32-channel head coil (Nova Medical, Wilmington, US). $\mathrm{B}_{0}$ shimming up to 3rd order was employed to minimize field inhomogeneity. The single-echo images were acquired using the CMRR SMS sequence implementation (release 11a) (https://www.cmrr.umn.edu/multiband/). The multi-echo acquisition used a SMS version of the multi-echo sequence described in Poser and Norris (2009). When choosing the sequence settings, the following key parameters were kept equal for both sequences to facilitate the comparison: spatial resolution (to prevent tSNR changes through differences in voxel volume), total scan time, in-plane acceleration factor using GRAPPA (Griswold et al., 2002) and slice acceleration factor using SMS (to obtain comparable image quality). SMS reconstruction was done using the slice-GRAPPA technique (Setsompop et al., 2012) as implemented in the CMRR sequence for the single-echo EPI, and the MGH blipped-CAIPI SMS-EPI sequence (https://www.nmr.mgh.harvard.edu/ software/c2p/sms) for the multi-echo sequence. The CMRR implementation for the single-echo sequence was chosen as it is a well-established, commonly used sequence for single-echo EPI (Van Essen et al., 2013). It also offers increased reconstruction speed compared to other sequences such as the single echo version of the multi-echo sequence. The in-plane acceleration factor was selected based on the multi-echo sequence to facilitate the acquisition of three echoes with sufficiently short echo times. Further shortening of the EPI echo trains was effected by use of phase partial Fourier (6/8) in the case of the multi-echo sequence. The slice acceleration factor was chosen to provide sub-second temporal resolution for whole-brain acquisition. Images were acquired with axial slices covering the brain from the most dorsal cortex to approximately midway through the cerebellum.

In detail, both sequences shared the following acquisition parameters: isotropic voxel size $=2.5 \mathrm{~mm}$, matrix size $=84 \times 84$, FOV $=212 \times 212 \mathrm{~mm}$, number of slices $=48$, flip angle $=35^{\circ}$, GRAPPA acceleration factor $=2$, SMS acceleration factor $=4$. The TE for the single-echo acquisition was set to $23 \mathrm{~ms}$, slightly shorter than the measured optimum for 7T BOLD fMRI in cortical areas (Berger et al., 2016; Koopmans et al., 2011; van der Zwaag et al., 2009; Yacoub et al., 2001). The three echoes of the multi-echo sequence were acquired at $9.9 \mathrm{~ms}, 28 \mathrm{~ms}$, and $45 \mathrm{~ms}$. While resolution and acceleration factors were equal for both sequences, the TR was set to the minimal possible length to maximize the number of acquired samples for a given run-length, resulting in a TR of $589 \mathrm{~ms}$ for the single-echo sequence and $830 \mathrm{~ms}$ for the multi-echo sequence.

For anatomical reference, a three-dimensional whole brain T1- weighted structural image was acquired for each participant using a prototype MP2RAGE sequence (WIP 900) (Marques et al., 2010; O'Brien et al., 2014) with the following parameters: $\mathrm{TE}=2.5 \mathrm{~ms}$, TR $=4300 \mathrm{~ms}$, flip angles $=5^{\circ}$ and $6^{\circ}$, inversion time $=840 \mathrm{~ms}$ and $2370 \mathrm{~ms}$, GRAPPA factor $=3$, acquisition matrix $=320 \times 300 \times 256$ and an isotropic voxel size of $0.75 \mathrm{~mm}$.

\section{Preprocessing}

All MRI data preprocessing was performed using the AFNI analysis package (Cox, 1996). Each functional EPI volume was registered to the volume with the minimum outlier fraction (i.e. the volume that is least different from all the others after detrending) to correct for head motion. The subject's anatomical images were aligned with the functional data by skull-stripping and then aligning the anatomical to the same EPI base (with minimum outlier fraction) using AFNI's align_epi_anat.py script. The functional data for each subject was transformed into a standard space by computing the transform required to non-linearly warp the subject's anatomical to match the Montreal Neurological Institute (MNI) 152 template at $0.75 \mathrm{~mm}$ isotropic resolution (Fonov et al., 2009) and then applying this tranform to the functional data.

The initial preprocessing steps were performed identically for both the single-echo and multi-echo data, with the only difference being that there were three sets of images per run for the multi-echo data rather than a single set per run as in the case of the single-echo data. As described, a weighted summation based on the temporal average was then used to combine the different echoes. To obtain estimates of T2* values in cortical and sub-cortical regions, we performed a voxel-wise linear fit on the logarithm across the three echoes for each subject on the temporal average, assuming a mono-exponential decay and low noise levels.

\section{Single-subject analysis}

All functional time-courses were scaled to percent signal change (i.e. each voxel time series was scaled to have a mean of 100) before calculating a multiple linear regression. The regression model contained 2 regressors corresponding to each experimental condition (simple and complex movement, Fig. 1C) generated by convolving the stimulus timing of each condition with a canonical hemodynamic response function model. A further 6 regressors, estimated from the volume registration step and representing the participant's head movement during the scan, were also included. From the calculated regression model, contrastto-noise ratio (CNR) was calculated on a voxel-wise basis for each condition by dividing each voxel's beta or linear contrast value from the general linear model analysis by the standard deviation of the residual error of the time-series for that voxel. Results from the single-subject regression analysis were then used in a second stage group analysis as described below.

In addition to the task-based fMRI, resting state scans were acquired for a single participant to assess the tSNR achieved with both sequences. To this end, 50 volumes were collected at rest using each sequence, and tSNR images were calculated by dividing the mean signal in each voxel by its standard deviation across all 50 volumes. The tSNR was then compared across regions of interest (ROIs) by calculating the average tSNR of all voxels within each ROI.

ROIs for the tSNR analysis included cortical regions M1 and SMA as well as the subcortical caudate nucleus (CN) and putamen (PUT) within the basal ganglia. The cortical regions were defined using the HarvardOxford atlas developed at the Center for Morphometric Analysis (CMA) and distributed with the FMRIB Software Library (FSL) (Desikan et al., 2006; Frazier et al., 2005; Goldstein et al., 2007; Makris et al., 2006). This probabilistic atlas was thresholded at $33 \%$ and resampled to match the resolution of the functional data (2.5 mm isotropic). For the subcortical nuclei, the basal ganglia were first identified using a recently published basal ganglia atlas derived from high-resolution 7T anatomical 
imaging (Keuken et al., 2014; Keuken and Forstmann, 2015). As this atlas defines the striatum as a single ROI, the striatum was then manually divided into the CN, PUT, and ventral striatum (VST) based on the guidelines set forth by Tziortzi et al. (2014).

\section{Group analysis}

To construct group statistical parametric maps to examine effects across all participants, mixed effects meta-analyses were performed using AFNI's 3dMEMA (Chen et al., 2012). This program models both withinand across-subject variability and therefore requires both the regression coefficients or a general linear contrast among them as well as the associated $t$-statistic from each subject. Being interested in the voxels that respond differentially to the complex vs. simple movement conditions, we used the general linear contrast between the two regression coefficients (complex - simple) and the corresponding $t$-statistics from each subject to construct the group maps.

The mixed effects meta-analysis was first performed separately for the single-echo and combined multi-echo data to construct sequence-specific group maps. The overall extent of activation detected using the different sequences was compared by counting the number of active voxels within each atlas-based ROI for each sequence-specific group map. Voxels were defined as active if they survived a threshold set to the false discovery rate (FDR)-corrected $q$ value of 0.05 (Genovese et al., 2002).

Next, those voxels within the atlas-based ROIs that were commonly activated in both sequences, i.e. those showing a significantly different response to the complex movement condition compared to the single movement condition across both sequences, were identified. To this end, data from both sequences were combined into one dataset and the same mixed effects meta-analysis was performed. The rationale for not including the entire structural ROIs was that only small regions within the relevant basal ganglia nuclei were expected to show activation due to the motor task (Bednark et al., 2015). Therefore, including all voxels within a structural ROI for analysis would result in a very small effect size, as the majority of voxels within the ROI would not show significant activation. These functionally-defined ROIs were then applied to the sequence-specific group maps to compare the $t$-statistic values measured using each sequence across an identical set of voxels. These same ROIs were also applied to group CNR maps constructed by voxel-wise averaging of the individual subject CNR maps.

\section{Results and discussion}

\section{tSNR in a single subject}

tSNR images were computed for each sequence using the resting state scans with an equal number of volumes from a single subject to illustrate basic signal properties (Fig. 2A) (Welvaert and Rosseel, 2013) and indicate the effect of weighting the first echo in the basal ganglia (Fig. 2B). Figure 2A (top) shows a single axial slice of the tSNR images computed for the single-echo data (left) and for the combined multi-echo data (right). The slice shown intersects the BG (red arrows), and as expected, although tSNR is high in the cortex, considerable signal loss can be seen in the basal ganglia in the single-echo data. The combined multi-echo data, however, suffers far less from this signal void, profiting from the increased signal levels incorporated from the earlier echo. This is illustrated in the tSNR images (Fig. 2A, bottom) and echo weighting maps (Fig. 2B) constructed separately for the individual echo data collected with the multi-echo sequence. Note the increased contribution of the first echo in the basal ganglia illustrated in the echo weighting maps.

To quantify the tSNR we averaged across all voxels in each ROI (Fig. 2C) finding that the multi-echo sequence yielded higher tSNR in all ROIs, both cortical and subcortical, with an average increase in tSNR of $84 \%$ in the basal ganglia ROIs. This was further supported by a pairedsample $t$-test performed on the tSNR values across all voxels within the ROIs, finding that the tSNR was significantly greater for the multi-echo sequence $(M=116, S D=42)$ than the single-echo sequence $(M=70$, $\mathrm{SD}=25) ; t(6835)=-114, p<0.001$. These results show that, as expected, tSNR of the multi-echo sequence is increased overall, partially due to a longer readout; making better use of the 'dead time' after the RF excitation is indeed one of the advantages of multi-echo EPI (Poser and Norris, 2009).

When optimizing both sequences, we aimed to keep key parameters, such as resolution, in-plane and slice acceleration factor, FOV and reconstruction technique (Setsompop et al., 2012), equal to facilitate the comparison and interpretation of the results. Note that the same flip angle was also used for both sequences despite different TRs. While image SNR strongly depends on the chosen flip angle with respect to the repetition time, tSNR remains nearly constant across a broad range of flip angles (Gonzalez-Castillo et al., 2011). Hence, we would not expect a noticeable impact on the tSNR measurements due to using the same flip angle at different TRs. Furthermore, reconstruction performance between the two sequences might differ slightly (e.g. g-factor related noise amplification), similar to when comparing the same sequence with different acceleration factors (Setsompop et al., 2012; Moeller et al., 2010). This is related to variations in the sequence implementation and the additional partial Fourier factor employed in the multi-echo acquisition, and hence, could not be fully avoided.

\section{Group statistical parametric mapping}

Group $t$-statistic maps were computed separately for the single-echo (Fig. 3A) and combined multi-echo (Fig. 3B) data to examine how the spatial distribution and extent of the detected activation compared between the two sequences. Bilateral activation was detected in each ROI for both sequences with the multi-echo sequence showing a greater extent of activation in all ROIs except the SMA (Fig. 3C). Qualitative comparison of the activation maps across sequences reveals the most striking difference is in the area of the basal ganglia (Fig. 3A,B - green arrows), with a much greater extent of activation and larger $t$-statistics evident in the multi-echo data. For example, we observed about a 3-fold increase in supra-threshold voxel in both the putamen and caudate nucleus for the multi-echo sequence (Fig. 3C). In addition to the a priori defined subcortical ROIs, significant bilateral activation was also detected in the thalamus (Fig. 3A,B - purple arrows). Although it is clear that a greater extent of activation was detected in the region of the thalamus using the multi-echo sequence compared to the single-echo, the responses in this region were not analyzed further. Recall that the group activation maps in Figure 3 were constructed using the contrast between the complex and simple movement conditions. Group activation maps for the two movement conditions vs. rest are included as supplementary material (Figs. S1 and S2) and, as expected, showed a much broader extent of cortical activation similar to that elicited by a conventional finger tapping motor paradigm.

\section{Comparing activation parameters}

To compare activation parameters across sequences and brain region, we extracted these from an identical set of voxels within each functionally-defined ROI (see Supplementary Fig. S3 for an illustration of the ROIs). A 2-way repeated measures ANOVA was performed to test the effect of sequence (single-echo, multi-echo) and ROI (M1, SMA, CN, PUT) on the magnitude of the $t$-statistic associated with the linear contrast between the two movement conditions (Fig. 4A). Mauchly's test revealed that the assumption of sphericity was violated so the Greenhouse-Geisser correction was used. A main effect was found for both sequence [F (1, $138)=111, \mathrm{p}<0.001)]$ and $\operatorname{ROI}[\mathrm{F}(2.5,340)=24, \mathrm{p}<0.001)]$ as well as significant interaction between the two $[\mathrm{F}(2.6,361)=46$, $\mathrm{p}<0.001)]$. The significant interaction indicates that the effect of the sequence was dependent on the ROI. As can be seen in Figure 4A, it is clear that the improvement in $t$-statistic provided by the multi-echo sequence is greater in subcortical compared to cortical areas, with one 
A.

tSNR Maps

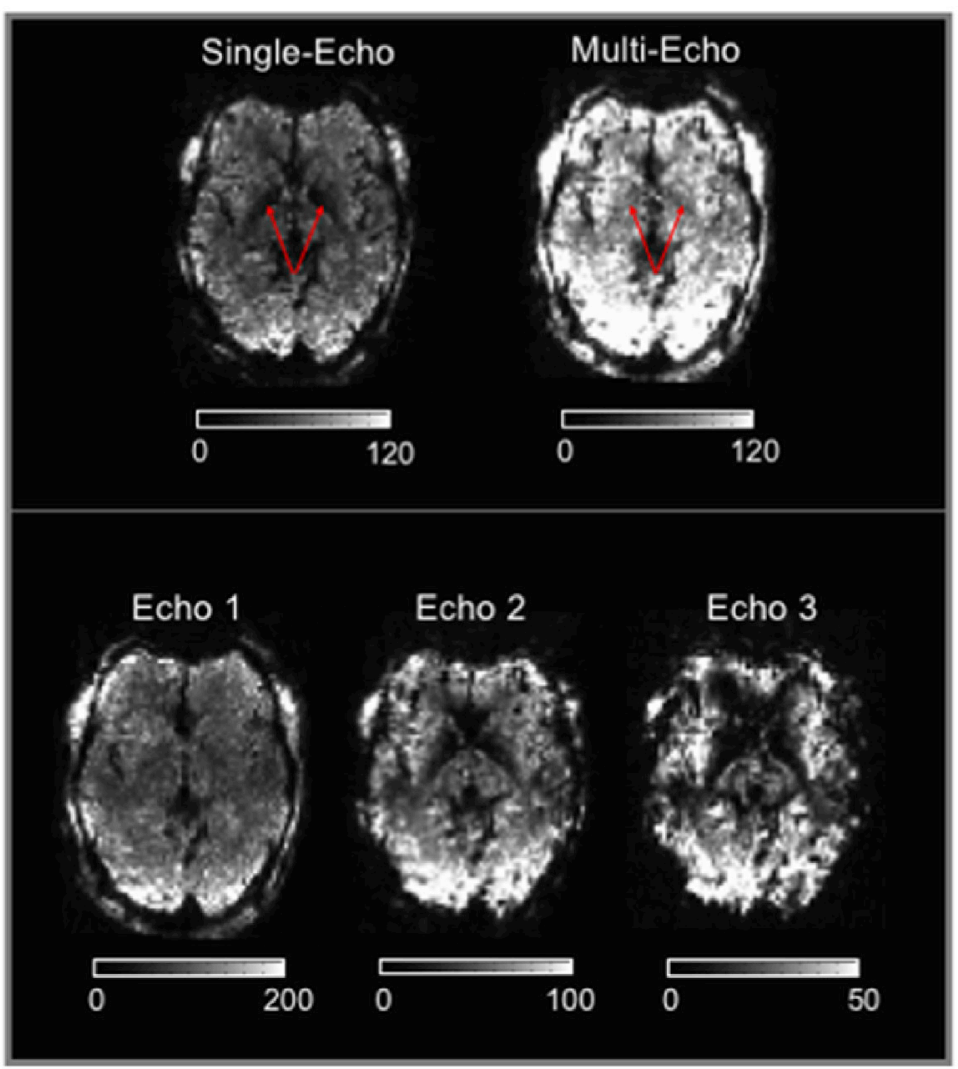

B.

Echo Weighting Maps

Echo 1

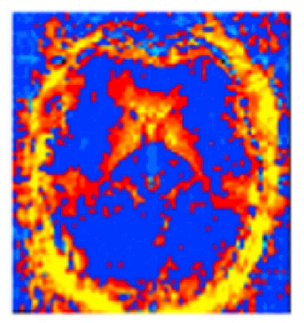

Echo 2

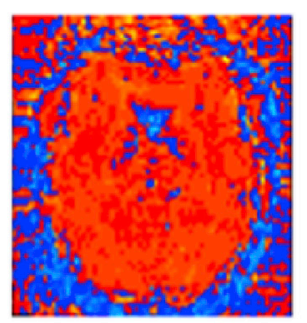

\section{Echo 3}
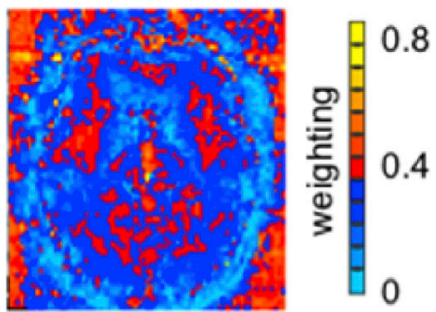

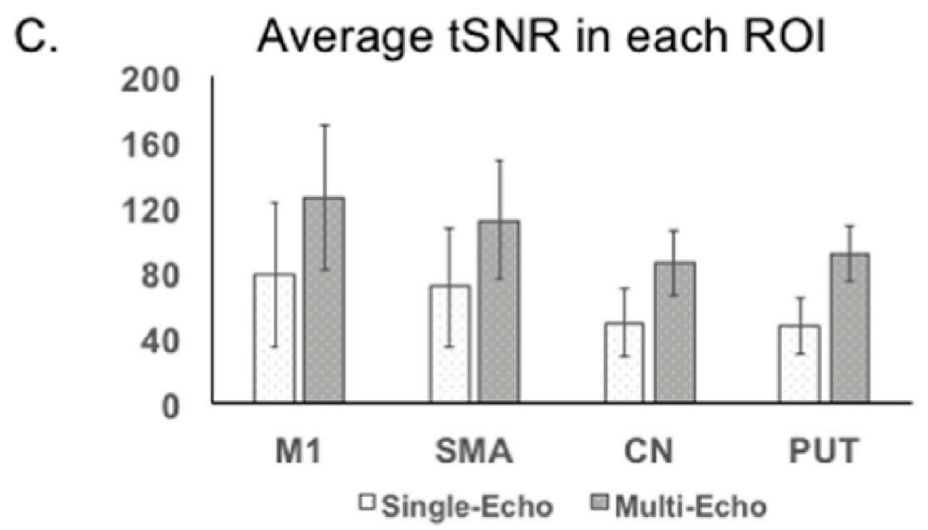

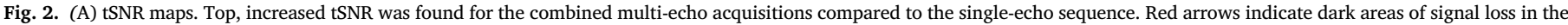

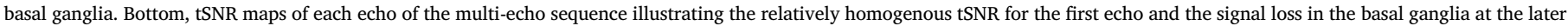

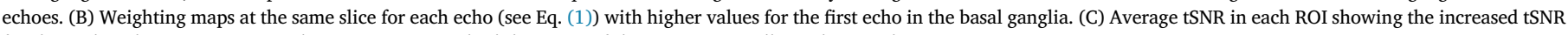
for the multi-echo sequence. Error bars represent standard deviation of the tSNR across all voxels in each ROI. 
A. Single-Echo Group Activation Map
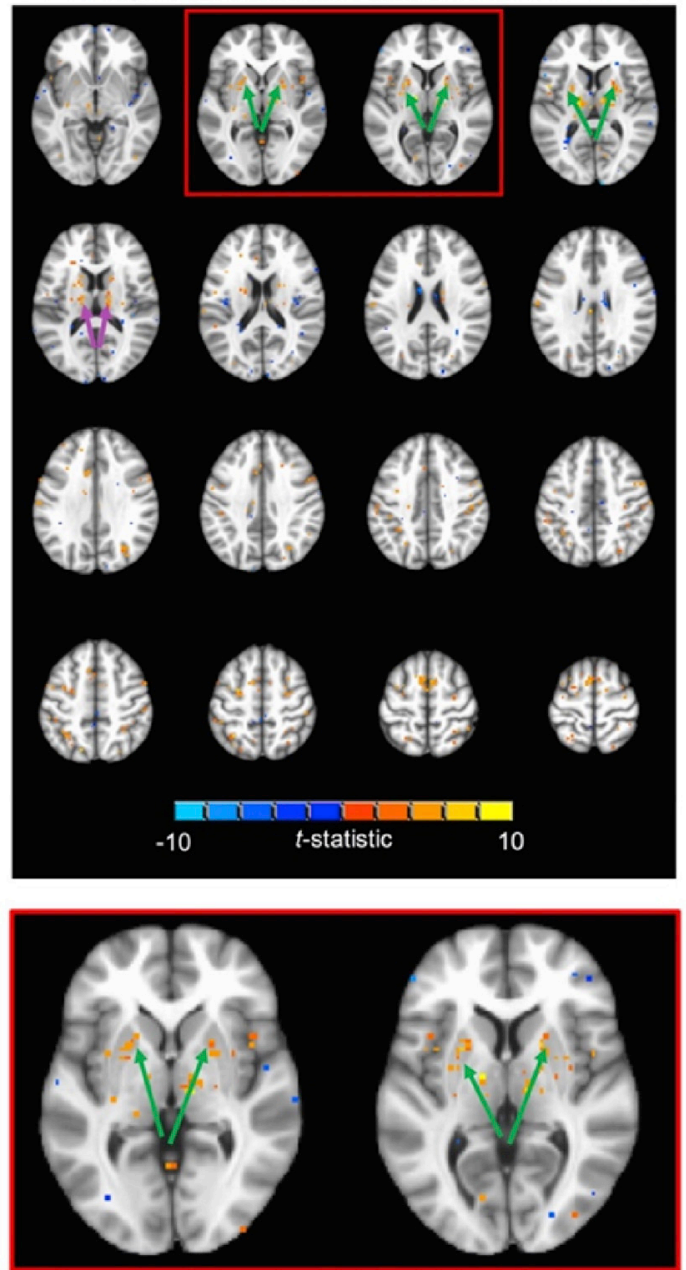

\section{B. Multi-Echo Group Activation Map}
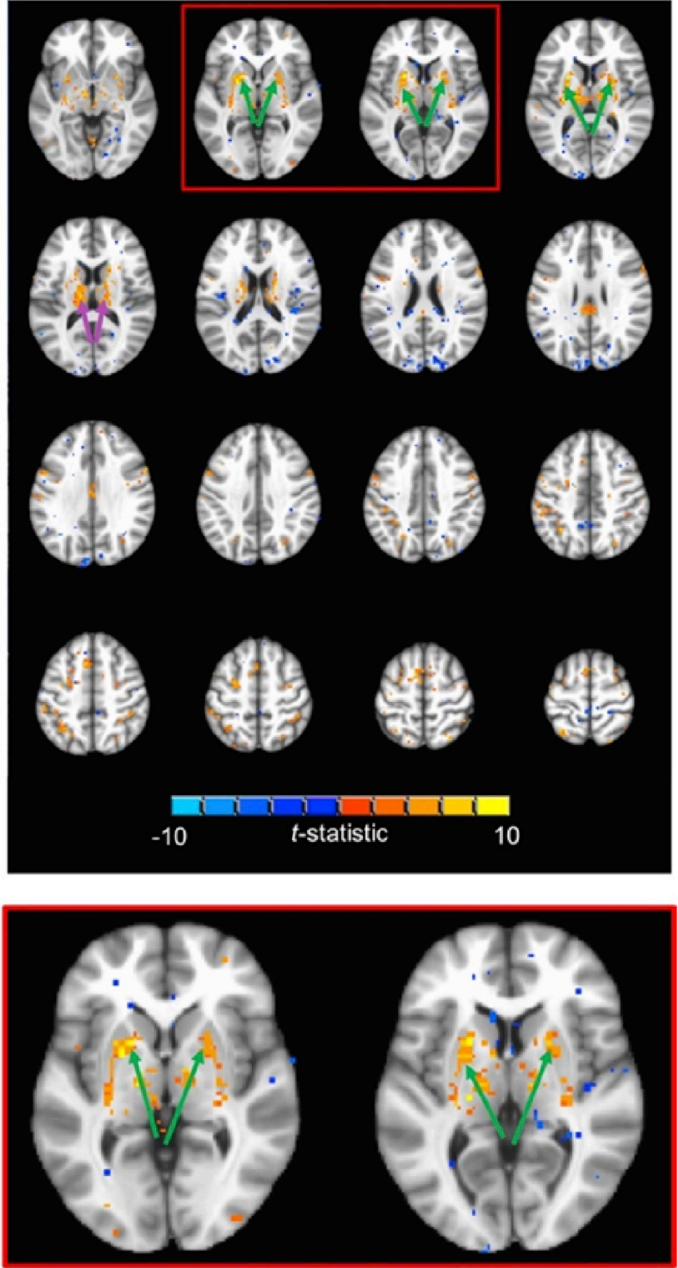

\section{Number of Active Voxels Detected}

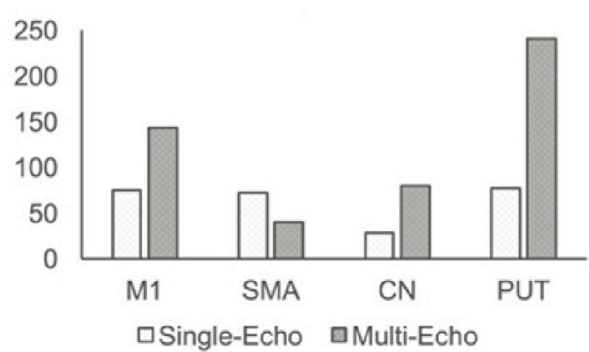

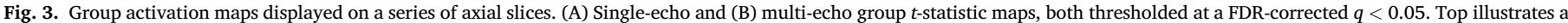

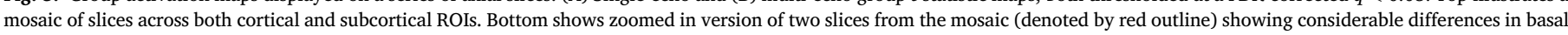

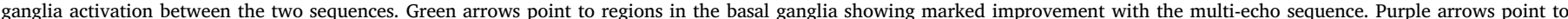

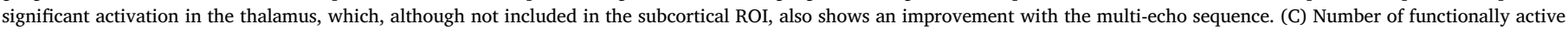
voxels detected (i.e. those surviving threshold) within each ROI.

of the cortical areas (SMA) actually showing a decrease in magnitude of the $t$-statistics when using multi-echo compared to single-echo data. When interpreting the $t$-statistics results here and in Figure 3 , it is important to note that, when computing statistical maps on the singlesubject level prior to performing the group analysis, the degrees-offreedom differed between the two sequences due to the single-echo sequence having a shorter TR (approximately 30\% more volumes were acquired using the single-echo sequence compared to the multi-echo sequence). Hence, the improvement seen using the multi-echo sequence is in spite of the advantage afforded by the faster TR of the single-echo sequence.

To better understand the improvement in $t$-statistic afforded by the multi-echo sequence, additional 2-way repeated measures ANOVAs were performed to test the effect of sequence (single-echo, multi-echo) and ROI (M1, SMA, CN, PUT) on the CNR (Fig. 4B). Again, a GreenhouseGeisser correction was used as Mauchly's test revealed that the assumption of sphericity was violated. A main effect was found for both sequence $[\mathrm{F}(1,138)=240, \mathrm{p}<0.001)]$ and ROI $[\mathrm{F}(2.2,305)=210, \mathrm{p}<0.001)]$ as well as a mild interaction between the two $[\mathrm{F}(2.0,280)=3.5$, $\mathrm{p}=0.03$ )]. Inspection of Figure $4 \mathrm{~B}$ reveals that the functional CNR was 
A.

t-statistic

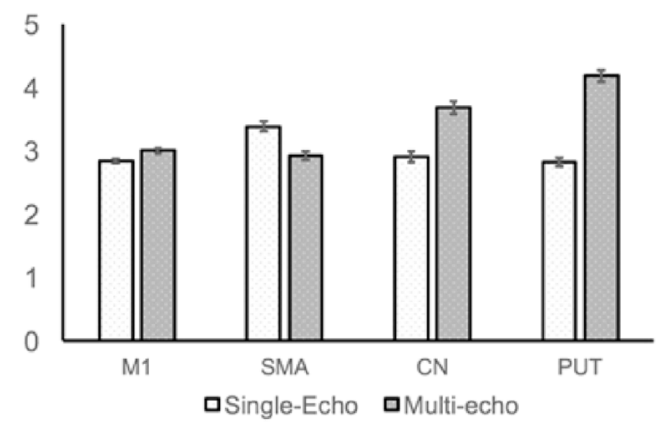

B.

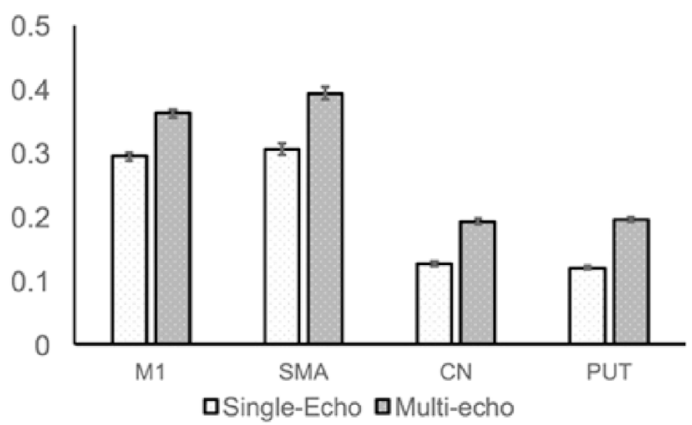

Fig. 4. Comparing group activation parameters across sequence and brain region. (A) Increase in mean $t$-statistic was observed in the putamen, caudate and in M1, with the highest improvement in the subcortical nuclei. (B) Increased CNR values were found in all ROIs. Error bars represent standard error of the mean.

significantly higher for the multi-echo compared to the single-echo data in all ROIs $(\mathrm{p}<0.001)$. Together, these results demonstrate that increase in CNR on the single-subject level can translate to an improvement in $t$ statistics on the group level, with higher $t$-value gains in the subcortical regions.

\section{Relationship of sensitivity gains to $\mathrm{T} 2$ *}

The main goal of this study was to investigate the potential benefit of using a multi-echo EPI sequence to improve the simultaneous imaging of cortical and subcortical areas with different underlying T2* values. From previous work, it was expected that multi-echo imaging would improve image quality across the whole-brain, however, it was also predicted that this improvement would be greater in iron-rich subcortical nuclei marked by lower T2* values compared to cortical areas. In the previous section, it was shown that improvement in $t$-statistic was indeed greater in subcortical areas compared to cortical regions. To further examine this issue, $\mathrm{T}^{*}$ values were estimated at each voxel and then compared to the degree of improvement in $t$-statistic provided by the multi-echo sequence. As expected, the average T2* varied across ROIs with the subcortical nuclei being marked by shorter T2* estimates than the cortical areas [ROI (mean,SD): M1 (25.2, 3.7); SMA (30.9, 9.7); CN (18.3, 2.4); PUT $(17.5,2.9)]$. Interestingly, these values are close to the $\mathrm{T} 2$ * values reported in (Peters et al., 2007), indicating that indeed the major source of signal dephasing in the basal ganglia are microscopic and mesoscopic susceptibility variations and not macroscopic field inhomogeneities. Figure 5 shows that there is a distinct correspondence between the $\mathrm{T} 2 *$ estimate and the improvement in $t$-statistic. It appears that the improvement is greatest in subcortical regions with lower T2* values relative to the cortical regions, with one of the cortical regions (SMA) actually showing a decrease in the average $t$-statistic when using the multi-echo sequence (Fig. 5). This relationship is further supported by a significant negative voxel-wise correlation between the T2* estimate and the improvement in $t$-statistic $(\mathrm{r}=-0.2665, \mathrm{P}<0.001)$.

\section{Conclusions and recommendations}

This study demonstrates that multi-echo imaging can be used to improve the concurrent functional imaging of the cortex and subcortex at 7T compared to conventional single-echo EPI. As predicted, the greatest improvement in detecting activation was seen in the iron-rich, subcortical nuclei of the basal ganglia. Importantly, this was achieved while maintaining sensitivity to functional activity in the cortex although a slight decrease in performance was seen in the supplementary motor area. The improvement afforded by multi-echo imaging was achieved by maximizing sensitivity to different echo times at each voxel through a weighted combination of the multiple echoes. By combining multi-echo imaging with an SMS acquisition scheme, it was possible to collect all three echoes with whole-brain coverage at a temporal resolution well below one second. Hence, the multi-echo SMS EPI sequence implemented here is well-suited to serve a wide range of functional imaging experiments.

One potential limitation for multi-echo EPI is the longer required echo train length. Using moderate parallel imaging factors and a conventional voxel size, we were, however, able to achieve sufficiently short TEs for imaging the iron rich basal ganglia nuclei at 7T. When higher resolution is required, one could either increase the acceleration factors of the parallel imaging methods employed here or resort to alternative acquisition schemes such as segmented Cartesian readouts (Hoogenraad et al., 2000; Li et al., 2002) or multi-echo spirals (Barth et al., 1999). Additionally, one could also further reduce the number of echoes, essentially acquiring only one early and one late echo. Given that the estimated average T2* in the Putamen was much higher than the first TE of the multi-echo sequence, it might be possible to increase the resolution and, thereby, lengthen the first TE and still obtain sufficient sensitivity in

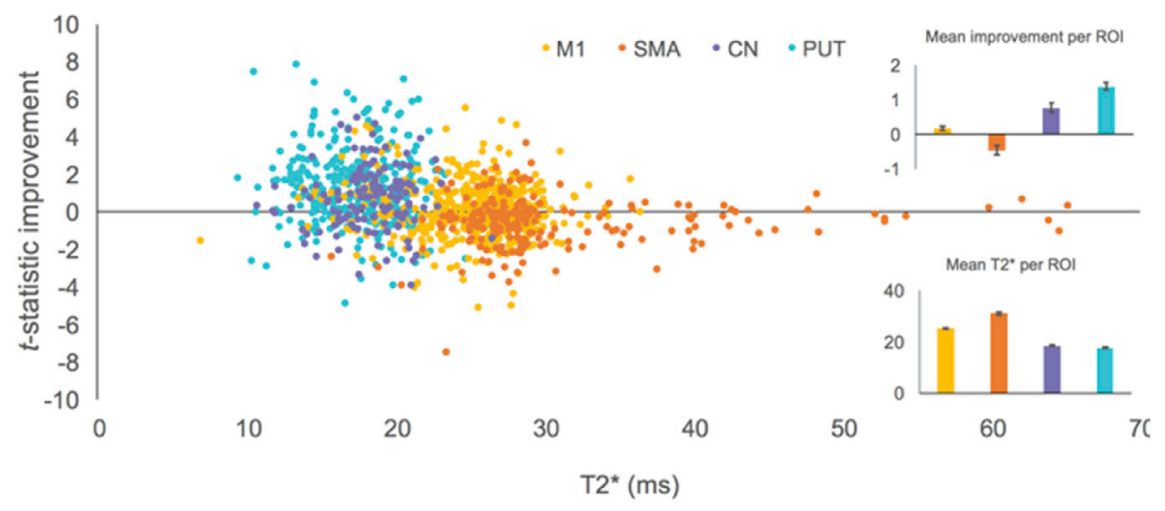

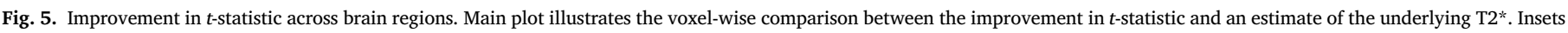
shows the average improvement (upper right) and T2* (lower right) in each ROI. Error bars represent standard error of the mean. 
the iron-rich basal ganglia nuclei.

While in this study the main focus was on increased sensitivity to the BOLD signal, multi-echo EPI-based techniques to reduce noise contributions from physiology are powerful means to further increase functional sensitivity. For example, MEICA has been shown to reliably detect and remove physiological noise from fMRI time series (Kundu et al., 2012, 2013). Physiological noise (i.e. signal fluctuations in fMRI time series associated with cardiac and respiratory activity (Weisskoff et al., 1993)) constitutes a major source of unwanted signal fluctuations (Bianciardi et al., 2009) increasing with field strength (Triantafyllou et al., 2005). Physiological noise is particularly problematic near the basal ganglia nuclei due to their proximity to major veins, with physiological noise modelling being shown to provide up to $30 \%$ tSNR gain in these subcortical nuclei at 3T (Kasper et al., 2017). In this respect, the results obtained here represent a lower bound on the boost in sensitivity provided by multi-echo EPI at ultra-high field, with a further potential increase in sensitivity and specificity through physiological noise modelling (Boyacioğlu et al., 2015).

We conclude that multi-echo EPI acquisition offers increased BOLD sensitivity in regions with short $\mathrm{T} 2 *$, such as the basal ganglia, and should be chosen over conventional GE-EPI when concurrently imaging cortical regions and iron-rich subcortical nuclei at 7T.

\section{Acknowledgments}

We thank Aiman Al-Najjar, Nicole Atcheson, and Steffen Bollmann for help with data collection. We thank Steve Cauley for providing a multiecho enabled variant of the MGH slice-GRAPPA reconstruction and Himanshu Bath for details on the sequence-recon interface. This research was supported by the National Health and Medical Research Council (APP 1088419). MB acknowledges funding from Australian Research Council Future Fellowship grant FT140100865. SB acknowledges support through the Australian Government Research Training Program Scholarship. The authors acknowledge the facilities of the National Imaging Facility (NIF) at the Centre for Advanced Imaging, University of Queensland.

\section{Appendix A. Supplementary data}

Supplementary data related to this article can be found at https://doi. org/10.1016/j.neuroimage.2017.12.005.

\section{References}

Abosch, A., Yacoub, E., Ugurbil, K., Harel, N., 2010. An assessment of current brain targets for deep brain stimulation surgery with susceptibility-weighted imaging at 7 tesla. Neurosurgery 67, 1745-1756 discussion 1756.

Barth, M., Metzler, A., Klarhofer, M., Roll, S., Moser, E., Leibfritz, D., 1999. Functional MRI of the human motor cortex using single-shot, multiple gradient-echo spiral imaging. Magn. Reson Imaging 17, 1239-1243.

Barth, M., Breuer, F., Koopmans, P.J., Norris, D.G., Poser, B.A., 2016. Simultaneous multislice (SMS) imaging techniques. Magn. Reson Imaging 75, 61-81.

Bednark, J.G., Campbell, M.E., Cunnington, R., 2015. Basal ganglia and cortical networks for sequential ordering and rhythm of complex movements. Front. Hum. Neurosci. 9, 421.

Berger, M.C., Bachert, P., Grobner, J., Nagel, A.M., 2016. Multi-contrast T2(*)relaxometry upon visual stimulation at 3T and 7T. Magn. Reson Imaging 34, 864-874.

Bianciardi, M., Fukunaga, M., van Gelderen, P., Horovitz, S.G., de Zwart, J.A., Shmueli, K., Duyn, J.H., 2009. Sources of functional magnetic resonance imaging signal fluctuations in the human brain at rest: a 7 T study. Magn. Reson Imaging 27, 1019-1029.

Boyacioğlu, R., Schulz, J., Koopmans, P.J., Barth, M., Norris, D.G., 2015. Improved sensitivity and specificity for resting state and task fMRI with multiband multi-echo EPI compared to multi-echo EPI at 7T. Neuroimage 119, 352-361.

Breuer, F.A., Blaimer, M., Heidemann, R.M., Mueller, M.F., Griswold, M.A., Jakob, P.M., 2005. Controlled aliasing in parallel imaging results in higher acceleration (CAIPIRINHA) for multi-slice imaging. Magn. Reson Med. 53, 684-691.

Brunheim, S., Johst, S., Pfaffenrot, V., Maderwald, S., Quick, H.H., Poser, B.A., 2017. Variable slice thickness (VAST) EPI for the reduction of susceptibility artifacts in whole-brain GE-EPI at 7 Tesla. Magn. Reson Mater Phy 1-17.
Chen, G., Saad, Z.S., Nath, A.R., Beauchamp, M.S., Cox, R.W., 2012. FMRI group analysis combining effect estimates and their variances. Neuroimage 60, 747-765.

Cohen-Adad, J., Polimeni, J.R., Helmer, K.G., Benner, T., McNab, J.A., Wald, L.L., Rosen, B.R., Mainero, C., 2012. T(2)* mapping and B(0) orientation-dependence at 7 T reveal cyto- and myeloarchitecture organization of the human cortex. Neuroimage $60,1006-1014$.

Cox, R.W., 1996. AFNI: software for analysis and visualization of functional magnetic resonance neuroimages. Comput. Biomed. Res. 29, 162-173.

Cunnington, R., Windischberger, C., Deecke, L., Moser, E., 2002. The preparation and execution of self-initiated and externally-triggered movement: a study of eventrelated fMRI. Neuroimage 15, 373-385.

Deistung, A., Schafer, A., Schweser, F., Biedermann, U., Turner, R., Reichenbach, J.R., 2013. Toward in vivo histology: a comparison of quantitative susceptibility mapping (QSM) with magnitude-, phase-, and R2*-imaging at ultra-high magnetic field strength. Neuroimage 65, 299-314.

Desikan, R.S., Segonne, F., Fischl, B., Quinn, B.T., Dickerson, B.C., Blacker, D. Buckner, R.L., Dale, A.M., Maguire, R.P., Hyman, B.T., Albert, M.S., Killiany, R.J., 2006. An automated labeling system for subdividing the human cerebral cortex on MRI scans into gyral based regions of interest. Neuroimage 31, 968-980.

Drayer, B., Burger, P., Darwin, R., Riederer, S., Herfkens, R., Johnson, G.A., 1986. MRI of brain iron. AJR Am. J. Roentgenol. 147, 103-110.

Van Essen, D.C., Smith, S.M., Barch, D.M., Behrens, T.E.J., Yacoub, E., Ugurbil, K., 2013. The WU-Minn human connectome project: an overview. Neuroimage 80, 62-79.

Feinberg, D.A., Moeller, S., Smith, S.M., Auerbach, E., Ramanna, S., Gunther, M., Glasser, M.F., Miller, K.L., Ugurbil, K., Yacoub, E., 2010. Multiplexed echo planar imaging for sub-second whole brain FMRI and fast diffusion imaging. PLoS One 5, e15710.

Fonov, V.S., Evans, A.C., McKinstry, R.C., Almli, C.R., Collins, D.L., 2009. Unbiased nonlinear average age-appropriate brain templates from birth to adulthood. Neuroimage 47, S102.

Frazier, J.A., Chiu, S., Breeze, J.L., Makris, N., Lange, N., Kennedy, D.N., Herbert, M.R., Bent, E.K., Koneru, V.K., Dieterich, M.E., Hodge, S.M., Rauch, S.L., Grant, P.E., Cohen, B.M., Seidman, L.J., Caviness, V.S., Biederman, J., 2005. Structural brain magnetic resonance imaging of limbic and thalamic volumes in pediatric bipolar disorder. Am. J. Psychiatry 162, 1256-1265.

Gati, J.S., Menon, R.S., Ugurbil, K., Rutt, B.K., 1997. Experimental determination of the BOLD field strength dependence in vessels and tissue. Magn. Reson Med. 38, 296-302.

Gelman, N., Gorell, J.M., Barker, P.B., Savage, R.M., Spickler, E.M., Windham, J.P., Knight, R.A., 1999. MR imaging of human brain at $3.0 \mathrm{~T}$ : preliminary report on transverse relaxation rates and relation to estimated iron content. Radiology 210, 759-767.

Genovese, C.R., Lazar, N.A., Nichols, T., 2002. Thresholding of statistical maps in functional neuroimaging using the false discovery rate. Neuroimage 15, 870-878.

Goldstein, J.M., Seidman, L.J., Makris, N., Ahern, T., O'Brien, L.M., Caviness Jr., V.S., Kennedy, D.N., Faraone, S.V., Tsuang, M.T., 2007. Hypothalamic abnormalities in schizophrenia: sex effects and genetic vulnerability. Biol. Psychiatry 61, 935-945.

Gonzalez-Castillo, J., Roopchansingh, V., Bandettini, P.A., Bodurka, J., 2011. Physiological noise effects on the flip angle selection in BOLD fMRI. Neuroimage 54, 2764-2778.

Griswold, M.A., Jakob, P.M., Heidemann, R.M., Nittka, M., Jellus, V., Wang, J., Kiefer, B., Haase, A., 2002. Generalized autocalibrating partially parallel acquisitions (GRAPPA). Magn. Reson Med. 47, 1202-1210.

Haacke, E.M., Xu, Y., Cheng, Y.C., Reichenbach, J.R., 2004. Susceptibility weighted imaging (SWI). Magn. Reson Med. 52, 612-618.

Haacke, E.M., Cheng, N.Y., House, M.J., Liu, Q., Neelavalli, J., Ogg, R.J., Khan, A., Ayaz, M., Kirsch, W., Obenaus, A., 2005. Imaging iron stores in the brain using magnetic resonance imaging. Magn. Reson Imaging 23, 1-25.

Hallgren, B., Sourander, P., 1958. The effect of age on the non-haemin iron in the human brain. J. Neurochem. 3, 41-51.

Hoogenraad, F.G., Pouwels, P.J., Hofman, M.B., Rombouts, S.A., Lavini, C., Leach, M.O., Haacke, E.M., 2000. High-resolution segmented EPI in a motor task fMRI study. Magn. Reson Imaging 18, 405-409.

Kasper, L., Bollmann, S., Diaconescu, A.O., Hutton, C., Heinzle, J., Iglesias, S., Hauser, T.U., Sebold, M., Manjaly, Z.M., Pruessmann, K.P., Stephan, K.E., 2017. The PhysIO toolbox for modeling physiological noise in fMRI data. J. Neurosci. Methods 276, 56-72.

Kettinger, A., Hill, C., Vidnyanszky, Z., Windischberger, C., Nagy, Z., 2016. Investigating the group-level impact of advanced dual-echo fMRI combinations. Front. Neurosci. 10, 571 .

Keuken, M.C., Forstmann, B.U., 2015. A probabilistic atlas of the basal ganglia using 7 T MRI. Data Brief. 4, 577-582.

Keuken, M.C., Bazin, P.L., Crown, L., Hootsmans, J., Laufer, A., Muller-Axt, C., Sier, R., van der Putten, E.J., Schafer, A., Turner, R., Forstmann, B.U., 2014. Quantifying interindividual anatomical variability in the subcortex using $7 \mathrm{~T}$ structural MRI. Neuroimage 94, 40-46.

Koopmans, P.J., Barth, M., Orzada, S., Norris, D.G., 2011. Multi-echo fMRI of the cortical laminae in humans at 7 T. Neuroimage 56, 1276-1285.

Kundu, P., Inati, S.J., Evans, J.W., Luh, W.M., Bandettini, P.A., 2012. Differentiating BOLD and non-BOLD signals in fMRI time series using multi-echo EPI. Neuroimage 60, 1759-1770.

Kundu, P., Brenowitz, N.D., Voon, V., Worbe, Y., Vertes, P.E., Inati, S.J., Saad, Z.S., Bandettini, P.A., Bullmore, E.T., 2013. Integrated strategy for improving functional connectivity mapping using multiecho fMRI. Proc. Natl. Acad. Sci. U. S. A. 110, 16187-16192. 
Kundu, P., Voon, V., Balchandani, P., Lombardo, M.V., Poser, B.A., Bandettini, P.A., 2017 Multi-echo fMRI: a review of appilcations in fMRI denoising and analysis of BOLD signals. Neuroimage 154, 59-80.

Larkman, D.J., Hajnal, J.V., Herlihy, A.H., Coutts, G.A., Young, I.R., Ehnholm, G., 2001. Use of multicoil arrays for separation of signal from multiple slices simultaneously excited. J. Magn. Reson Imaging 13, 313-317.

Lehericy, S., Bardinet, E., Tremblay, L., Van de Moortele, P.F., Pochon, J.B., Dormont, D., Kim, D.S., Yelnik, J., Ugurbil, K., 2006. Motor control in basal ganglia circuits using fMRI and brain atlas approaches. Cereb. Cortex 16, 149-161.

Li, Z., Wu, G., Zhao, X., Luo, F., Li, S.J., 2002. Multiecho segmented EPI with z-shimmed background gradient compensation (MESBAC) pulse sequence for fMRI. Magn. Reson Med. 48, 312-321.

Makris, N., Goldstein, J.M., Kennedy, D., Hodge, S.M., Caviness, V.S., Faraone, S.V., Tsuang, M.T., Seidman, L.J., 2006. Decreased volume of left and total anterior insular lobule in schizophrenia. Schizophr. Res. 83, 155-171.

Marques, J.P., Kober, T., Krueger, G., van der Zwaag, W., Van de Moortele, P.F., Gruetter, R., 2010. MP2RAGE, a self bias-field corrected sequence for improved segmentation and T1-mapping at high field. Neuroimage 49, 1271-1281.

Moeller, S., Yacoub, E., Olman, C.A., Auerback, E., Strupp, J., Harel, N., Ugurbil, K., 2010. Multiband multislice GE-EPI at 7 tesla, with 16-fold acceleration using partial parallel imaging with application to high spatial and temporal whole-brain fMRI. Magn. Reson Med. 63, 1144-1153.

O'Brien, K.R., Kober, T., Hagmann, P., Maeder, P., Marques, J., Lazeyras, F., Krueger, G., Roche, A., 2014. Robust T1-weighted structural brain imaging and morphometry at 7T using MP2RAGE. PLoS One 9, e99676.

Ordidge, R.J., Gorell, J.M., Deniau, J.C., Knight, R.A., Helpern, J.A., 1994. Assessment of relative brain iron concentrations using T2-weighted and T2*-weighted MRI at 3 Tesla. Magn. Reson Med. 32, 335-341.

Peters, A.M., Brookes, M.J., Hoogenraad, F.G., Gowland, P.A., Francis, S.T., Morris, P.G., Bowtell, R., 2007. T2* measurements in human brain at 1.5, 3 and 7 T. Magn. Reson Imaging 25, 748-753.

Poser, B.A., Norris, D.G., 2009. Investigating the benefits of multi-echo EPI for fMRI at 7 T. Neuroimage $45,1162-1172$

Poser, B.A., Versluis, M.J., Hoogduin, J.M., Norris, D.G., 2006. BOLD contrast sensitivity enhancement and artifact reduction with multiecho EPI: parallel-acquired inhomogeneity-desensitized fMRI. Magn. Reson Med. 55, 1227-1235.

Posse, S., Wiese, S., Gembris, D., Mathiak, K., Kessler, C., Grosse-Ruyken, M.L. Elghahwagi, B., Richards, T., Dager, S.R., Kiselev, V.G., 1999. Enhancement of BOLDcontrast sensitivity by single-shot multi-echo functional MR imaging. Magn. Reson Med. 42, 87-97.

Robinson, S., Windischberger, C., Rauscher, A., Moser, E., 2004. Optimized 3 T EPI of the amygdalae. Neuroimage 22, 203-210.

Robinson, S.D., Pripfl, J., Bauer, H., Moser, E., 2008. The impact of EPI voxel size on SNR and BOLD sensitivity in the anterior medio-temporal lobe: a comparative group study of deactivation of the Default Mode. MAGMA 21, 279-290.

Schafer, A., Forstmann, B.U., Neumann, J., Wharton, S., Mietke, A., Bowtell, R., Turner, R., 2012. Direct visualization of the subthalamic nucleus and its iron distribution using high-resolution susceptibility mapping. Hum. Brain Mapp. 33, 2831-2842.
Schenck, J.F., 2003. Magnetic resonance imaging of brain iron. J. Neurol. Sci. 207, 99-102.

Schweser, F., Deistung, A., Lehr, B.W., Reichenbach, J.R., 2011. Quantitative imaging of intrinsic magnetic tissue properties using MRI signal phase: an approach to in vivo brain iron metabolism? Neuroimage 54, 2789-2807.

Sedlacik, J., Boelmans, K., Lobel, U., Holst, B., Siemonsen, S., Fiehler, J., 2014. Reversible, irreversible and effective transverse relaxation rates in normal aging brain at 3T. Neuroimage 84, 1032-1041.

Setsompop, K., Gagoski, B.A., Polimeni, J.R., Witzel, T., Wedeen, V.J., Wald, L.L., 2012. Blipped-controlled aliasing in parallel imaging for simultaneous multislice echo planar imaging with reduced g-factor penalty. Magn. Reson Med. 67, 1210-1224.

Speck, O., Hennig, J., 1998. Functional imaging by I0- and T2*-parameter mapping using multi-image EPI. Magn. Reson Med. 40, 243-248.

Stocker, T., Kellermann, T., Schneider, F., Habel, U., Amunts, K., Pieperhoff, P., Zilles, K., Shah, N.J., 2006. Dependence of amygdala activation on echo time: results from olfactory fMRI experiments. Neuroimage 30, 151-159.

Triantafyllou, C., Hoge, R.D., Krueger, G., Wiggins, C.J., Potthast, A., Wiggins, G.C., Wald, L.L., 2005. Comparison of physiological noise at 1.5 T, 3 T and $7 \mathrm{~T}$ and optimization of fMRI acquisition parameters. Neuroimage 26, 243-250.

Tziortzi, A.C., Haber, S.N., Searle, G.E., Tsoumpas, C., Long, C.J., Shotbolt, P., Douaud, G., Jbabdi, S., Behrens, T.E., Rabiner, E.A., Jenkinson, M., Gunn, R.N., 2014. Connectivity-based functional analysis of dopamine release in the striatum using diffusion-weighted MRI and positron emission tomography. Cereb. Cortex 24, 1165-1177.

van der Zwaag, W., Francis, S., Head, K., Peters, A., Gowland, P., Morris, P., Bowtell, R., 2009. fMRI at 1.5, 3 and 7 T: characterising BOLD signal changes. Neuroimage 47, 1425-1434.

Volz, S., Hattingen, E., Preibisch, C., Gasser, T., Deichmann, R., 2009. Reduction of susceptibility-induced signal losses in multi-gradient-echo images: application to improved visualization of the subthalamic nucleus. Neuroimage 45, 1135-1143.

Weiskopf, N., Hutton, C., Josephs, O., Deichmann, R., 2006. Optimal EPI parameters for reduction of susceptibility-induced BOLD sensitivity losses: a whole-brain analysis at $3 \mathrm{~T}$ and $1.5 \mathrm{~T}$. Neuroimage 33, 493-504.

Weiskopf, N., Hutton, C., Josephs, O., Turner, R., Deichmann, R., 2007. Optimized EPI for fMRI studies of the orbitofrontal cortex: compensation of susceptibility-induced gradients in the readout direction. MAGMA 20, 39-49.

Weisskoff, R.M., Baker, J., Belliveau, J., Davis, T.L., Kwong, K.K., Cohen, M.S., Rosen, B.R., 1993. Power spectrum analysis of functionally-weighted MR data: what's in the noise? Proc. ISMRM $1,7$.

Welvaert, M., Rosseel, Y., 2013. On the definition of signal-to-noise ratio and contrast-tonoise ratio for fMRI data. PLOS ONE 8 (11).

Yablonskiy, D.A., 1998. Quantitation of intrinsic magnetic susceptibility-related effects in a tissue matrix. Phantom study. Magn. Reson Med. 39, 417-428.

Yacoub, E., Shmuel, A., Pfeuffer, J., Van De Moortele, P.F., Adriany, G., Andersen, P., Vaughan, J.T., Merkle, H., Ugurbil, K., Hu, X., 2001. Imaging brain function in humans at 7 Tesla. Magn. Reson Med. 45, 588-594.

Yao, B., Li, T.Q., Gelderen, P., Shmueli, K., de Zwart, J.A., Duyn, J.H., 2009. Susceptibility contrast in high field MRI of human brain as a function of tissue iron content. Neuroimage 44, 1259-1266. 